\title{
PRINCIPIO DE DISCURSO Y DEMOCRACIA DIRECTA: FAKTIZITÄT UND GELTUNG Y LA OBRA DE B. ACKERMAN*
}

\author{
A. Abad \\ Universidad de Buffalo \\ J. Monserrat \\ Universitat de Barcelona
}

\begin{abstract}
RESUMEN. Destacamos los elementos de la obra de B. ACKERMAN que pueden mejorar el concepto de democracia deliberativa dirigida a la legitimación de las normas jurídicas propuesta por HABERMAS en Faktizität und Geltung en relación a los principios de democracia deliberativa, el principio normativo, el principio de universalidad, el principio de igualdad, el concepto de esfera pública y el concepto de ciudadanía. Esta complementación busca dotar de más elementos el proyecto ideal Habermasiano para consolidarse como un marco a seguir en la codificación de los derechos. Mediante la obra de B. ACKERMAN intentamos aportar algo a los denominados espacios vacíos que deja HABERMAS entre el discurso sobre principios universales de justificación racional y los sentidos de la vida histórica concreta, el paso de los problemas de interpretación, enjuiciamiento y aplicación normativa. Consideramos que la obra de ACKERMAN puede suponer una excelente contribución al proceso de democracia deliberativa y a la acción comunicativa iniciada por HABERMAS y a las teorías contractualistas desde una perspectiva liberal.
\end{abstract}

Palabras clave: democracia deliberativa, acción comunicativa, teorías contractualistas.

* Fecha de recepción: 8 de mayo de 2008. Fecha de aceptación: 8 de julio de 2008.

Partimos en este artículo de los argumentos desplegados en A. ABAD y J. MONSERRAT, «Habermas and Ackerman, a synthesis of their Thought applied to the legitimation and codification of legal norms», en Ratio Juris, 3, 2009, in print, y «Monism Versus Dualism in the current Spanish System», en International Review of Constitutionalism, 8, 2008, pp. 1-40. Forma parte del Proyecto de Investigación financiado por la Dirección General de Investigación del Ministerio de Educación y Ciencia HUM2007-62763/FISO. B. ACKERMAN es profesor de Derecho y Ciencias Políticas en la Universidad Sterling de Yale desde 1987. Sus campos de estudio son el Derecho constitucional, la filosofía política y legal, el Derecho del medio ambiente, el Derecho de la propiedad y el Derecho tributario. El profesor ACKERMAN antes de acceder a su actual posición fue profesor de Derecho público y análisis político en la Universidad de Pennsylvania (1973-1974), profesor de Derecho y Filosofía en la Charles Keller Beekman, Columbia (1982-1987). El profesor ACKERMAN está considerado como uno de los pensadores actuales más importantes en teoría política y constitucional de los Estados Unidos. El esquema de trabajo que ha usado para desarrollar su teoría ha partido de un análisis muy depurado de la historia constitucional y política de los Estados Unidos. ACKERMAN analiza los momentos «constitucionales» que ha sufrido la historia constitucional norteamericana y extrae conclusiones y consecuencias que son de aplicabilidad en la actualidad. Estas conclusiones afectan de manera directa la legitimación del Derecho, la interpretación de la Constitución y las formas de participación democrática de los ciudadanos, tanto a nivel político como a nivel jurídico. Ha trabajado en varios proyectos constitucionales en diferentes partes del mundo y en la actualidad trabaja en los efectos a nivel jurídico de las decisiones políticas que toman los gobiernos en momentos de emergencia nacional, como podría ser ante atentados terroristas, etcétera. 
ABSTRACT. We highlight the elements of B. ACKERMAN's work that can improve the concept of deliberative democracy addressed to the legitimacy of norms proposed by HABERMAS in Faktizität und Geltung in relation to the principles of deliberative democracy, the normative principle, the principle of universality, the principle of equality, the concept of public sphere and the concept of citizenship. This enhancement seeks to provide more elements to the Habermasian ideal project to consolidate it as a framework to be followed in the right's codification. Through the work of $\mathrm{B}$. ACKERMAN we try to add something to the so-called empty spaces that HABERMAS left between the discourse on universal principles of rational justification and the meanings of concrete historical life, the passage of the problems of interpretation, judgment and rules' enforcement. We believe that ACKERMAN's work can formulate an excellent contribution to the process of deliberative democracy and communicative action initiated by HABERMAS and to the contract theories from a liberal perspective.

Keywords: deliberative democracy, communicative action, contractualist theories.

\section{INTRODUCCIÓN}

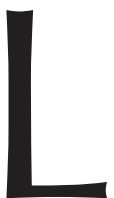

a aplicación de la democracia deliberativa en el campo del Derecho en la obra de J. HABERMAS Faktizität und Geltung, puede completarse con conceptos que B. ACKERMAN ha trabajado desde la experiencia histórica constitucional y política de los Estados Unidos. Partimos de una serie de similitudes básicas entre las teorías de ambos autores. En este sentido, los dos proponen un nuevo examen de las constituciones contemporáneas y de su sistema constituyente centrándose en diferentes tipos de justificación democrática, pero ambos autores ofrecen un modelo procedimental de legitimación democrática, otorgada mediante la deliberación pública. Con la exposición de los elementos de la obra de ACKERMAN analizaremos en qué concepto de la obra de HABERMAS se pueden aplicar, y por qué con la complementación de ambas teorías se mejora el proyecto de democracia deliberativa. Aspectos como la diferenciación de los momentos constitucionales y normales, los conceptos de diálogo político y jurídico y sus similitudes y diferencias, la consideración necesaria de la segunda generación, los principios de igualdad y de universalidad y las condiciones mínimas necesarias para ser considerado un ciudadano, pueden enriquecer el principio democrático y de discurso de HABERMAS. La realización de estas propuestas de mejora será posible si nos situamos en una perspectiva filosófica que permita abrazar y superar las implicaciones jurídico-políticas de ambas propuestas.

En el siguiente apartado («Concepto de diálogo neutro»), veremos cómo ACKERMAN, a diferencia de HABERMAS, considera que el diálogo entre ciudadanos miembros de una sociedad liberal es un diálogo constrictivo, es decir, los ciudadanos mediante el diálogo intentan convencer a los otros miembros de la sociedad de manera competitiva y no de forma neutral y basada en argumentos estrictamente racionales. ACKERMAN introduce el concepto de diálogo neutro cuando analiza el diálogo en las sociedades liberales modernas y realiza un tipo de depuración de esta «constrictiveness». Recordaremos cómo HABERMAS, en Teoría de la Acción Comunicativa, no analiza esta característica del diálogo en las sociedades liberales y se limita a afirmar que las personas dialogan en base a principios racionales, sin determinar los caracteres específicos del diálogo en el ámbito jurídico. El diálogo es, además, la base de los principios democráticos y de discurso sobre los cuales HABERMAS basa la legitimidad de las normas 
jurídicas. ACKERMAN acota en el tiempo el diálogo puesto que HABERMAS considera que las personas pueden mantener el diálogo indefinidamente hasta que la decisión se tome por unanimidad entre todos los posibles afectados por la norma.

En el tercer apartado («Momentos normales y momentos constitucionales»), veremos como ACKERMAN realiza un análisis político y jurídico de la historia constitucional americana. Consideramos que la introducción de la experiencia revolucionaria americana en el pensamiento político europeo es necesaria para comprender el alcance de la política contemporánea. Los momentos constitucionales son aquellos momentos en que las personas se ven afectadas por situaciones «anormales» que afectan de manera directa su estado anímico y psicológico de ciudadano normal y su capacidad de diálogo. Momentos constitucionales, como momentos de «suspensión-fundación», son, a lo largo de la historia de los Estados Unidos, el momento inicial de constitución, las campañas federalistas y el New Deal. Entender y preparar el proceso legislativo en estos momentos imprevisibles pero no imposibles es aquello que pretende ACKERMAN, pues sucede que a pesar de que son los momentos en que se establecen los cambios fundamentales que afectan a la misma naturaleza de la sociedad política, los ciudadanos pueden no estar capacitados entonces para legislar.

En contraposición a estos momentos constitucionales, encontramos los momentos denominados «normales», donde los ciudadanos no se ven afectados por acontecimientos extraordinarios; son aquellos que se producen de manera cotidiana. Es en este espacio de tiempo donde los ciudadanos tienen que regular, en la medida de lo posible, los efectos jurídicos y políticos que se producirán en los momentos constitucionales. En los momentos normales los ciudadanos no ven afectada su capacidad de diálogo final, a pesar de que el resultado de la normalidad puede ser una «neutralización» de la política en manos de los agentes representativos (partidos y clase política). ACKERMAN propone que en los momentos normales se regule de forma provisoria cómo actuar en los momentos constitucionales.

En el cuarto apartado («Democracia dualista»), exponemos cómo ACKERMAN defiende un tipo de Constitución dualista, que es aquella que busca la diferenciación entre los dos diferentes órganos de decisión sobre los que se produce una democracia. La primera decisión es tomada por el pueblo y, la segunda, por sus gobernantes. La definición de ACKERMAN analiza cómo el pueblo de los Estados Unidos ha decidido en momentos constitucionales a lo largo de la historia en contra de sus gobernantes, en claro ejemplo práctico e histórico de este tipo de democracia dualista. La democracia dualista mejora, según ACKERMAN, el concepto de democracia monista (aquella en la que son los gobernantes los únicos que deciden), puesto que introduce más democracia directa en la toma de decisiones. ACKERMAN introduce este concepto de democracia dualista en nombre del realismo y no en base a un tipo de idealismo utópico y lo sitúa en el centro del sistema democrático. El análisis del sistema dualista es importante en relación con nuestro trabajo porque nos permite analizar cómo actúan los ciudadanos respecto a la legitimación de las normas. Dentro de la misma definición del sistema de democracia dualista encontramos uno de los aspectos más transcendentes de la obra de ACKERMAN, a saber, la definición de «Pueblo» y de «esfera pública» (ACKERMAN, 1993). Esta definición debe complementar el principio de legitimación democrática de normas jurídicas en HABERMAS. 
Pretendemos mostrar también un grado más de concreción en el tema que planteamos con una serie de ejemplos. HABERMAS, en Faktizität und Geltung, elude este punto fundamental cuando define su principio democrático de legitimación de normas jurídicas. En consecuencia, los ciudadanos se verían vinculados por el contenido de normas jurídicas allí donde no han podido participar en su codificación. ACKERMAN ha realizado conjuntamente con FISHKIN una propuesta democrática en base a experiencias empíricas realizadas (B. ACKERMAN y J. FISHKIN, 2004). Uno de los aspectos más destacables del «Deliberation Day» es ver cómo se aplicaría este experimento político desde una perspectiva práctica. Los autores consideran cuestiones de carácter económico, político y organizativo relacionadas con el concepto de democracia liberal y su puesta en práctica, como por ejemplo incentivos económicos y laborales para los ciudadanos en el momento de participar en el proceso de decisión durante el día de deliberación. Asimismo, ACKERMAN analiza cómo las segundas generaciones afectan al diálogo neutro y cómo las decisiones tomadas por los ciudadanos liberales afectan a las generaciones posteriores que no han participado en tal toma de decisión. ACKERMAN, además de plantear esta omisión de los pensadores liberales, establece un sistema que posibilita esta necesaria participación de las siguientes generaciones en relación al contenido de la norma que les será aplicada como consecuencia de un proceso de legitimación democrática deliberativa directa. La aplicación al sistema de legitimación democrática de las normas jurídicas de la corrección, en relación a las segundas generaciones extiende la legitimación democrática necesaria entre las diferentes generaciones. Otro elemento que mencionaremos será la propuesta que realiza ACKERMAN en relación a una nueva separación de poderes. Esta separación no se limita a los tres poderes convencionales sino que se añaden otros dentro de lo que el autor denomina «The integrity Branch» $\mathrm{y}$ «The regulatory Branch» (ACKERMAN, 2000, pp. 633-727). ACKERMAN analiza cómo afecta la nueva separación propuesta a los derechos fundamentales y a los principios de justicia distributiva. El análisis parte de un completo estudio de los diferentes sistemas democráticos unicamerales y bicamerales. $\mathrm{El}$ autor, además, realiza un ataque al problema de la burocracia que afecta a los poderes del Estado y cómo este problema afecta a las decisiones y acciones finales de estos poderes. El problema de la burocracia también se omite en el sistema de legitimación normativa de HABERMAS.

Nos centramos en analizar, pues, los aspectos que entendemos que mejor complementan ambas teorías y que nos permitirán complementar la teoría de legitimación de normas jurídicas, complementación que a pesar de ser ideal, como ambas teorías en origen, puede ser un marco de referencia en el campo de la legitimación práctica de las normas jurídicas.

\section{CONCEPTO DE DIÁLOGO NEUTRO}

ACKERMAN, como HABERMAS, otorga una importancia especial al concepto y funciones del diálogo en el conjunto de su obra. El diálogo es la primera obligación que adquirimos como ciudadanos y resulta ser uno de los elementos nucleares de las sociedades liberales. Éstas deben posibilitar espacios donde se pueda practicar el diálo- 
go público entre los ciudadanos (ACKERMAN, 1989, p. 6) ${ }^{1}$. Además de las funciones sociales que tiene el concepto de diálogo, él mismo es un elemento fundamental para nuestra autodefinición moral, puesto que es la herramienta mediante la cual podemos tener en consideración los otros conciudadanos ${ }^{2}$.

ACKERMAN parte de la base que el diálogo entre ciudadanos de una sociedad liberal es de tipo constrictivo, es decir, los ciudadanos mediante el diálogo intentan convencer a los otros miembros de la sociedad de una manera competitiva y no de forma neutral, basada en argumentos estrictamente racionales. Esta es una característica definitoria del concepto de diálogo en ACKERMAN. HABERMAS, en su concepto de diálogo, parte de un tipo de diálogo plano, sujeto a principios argumentales racionales. HABERMAS, en Faktizität und Geltung, no analiza cómo es el diálogo que debe aplicarse al Derecho, sino que meramente lo somete al filtro de la racionalidad.

ACKERMAN analiza cómo es el diálogo y las funciones que tiene en una sociedad. Entre estas funciones destaca el hecho de que el diálogo sirve para controlar de manera sensible el poder de represión que se puede ejercer en una sociedad por parte de determinados estratos sociales. Considera que el diálogo es central en la vida pública, a pesar de que la práctica política no sea el lugar más idóneo para buscar la verdad moral mediante el diálogo. Con independencia de este handicap, el diálogo sirve para otras cuestiones transcendentales, como el hecho de organizar la vida en conjunto incluyendo las personas que están en desacuerdo sobre la verdad moral determinada (ACKERMAN, 1989, p. 8).

El establecimiento de la verdad moral, tanto a nivel individual como colectivo, es algo determinante a la hora de poner en práctica los marcos legales que regularán el Estado o determinadas normas que deben servir de principios generales del Derecho. En consecuencia, este procedimiento tiene una trascendencia directa y relevante en el proceso de aprobación de las normas jurídicas y de principios generales del Derecho. ACKERMAN afirma que como mejor se entiende la tradición liberal es justamente con el esfuerzo de definir y justificar una fuerza en el poder del habla. La noción de conversación constrictiva puede ser considerada como el principio organizador del pensamiento liberal (ACKERMAN, 1980, p. 10) 3 . La conversación política en el Estado liberal es un aparato necesario para organizar personas, las cuales son libres de entender de varias maneras el concepto del bien (ACKERMAN, 1980, p. 359). La idea de conversación

${ }^{1}$ B. ACKERMAN y J. S. FISHKIN, 2003, p. 8, dice: «We have a public dialogue that is ever more efficiently segmented in its audiences and morselized in its sound bites. We have had ever more tabloid news agenda dulling the sensitivities increasingly inattentive citizenry. And we have mechanisms of feedback from the public, from viewer call-ins to self-selected internet polls that emphasize intense constituencies, unrepresentative of public at large. If we have to preserve and deepen our democratic life, we must take the future into our own hands. We must create institutions that sustain citizen engagement in a shared public dialogue».

2 B. ACKERMAN, 1989, pp. 5-22. El autor afirma que el valor moral de su vida no depende exclusivamente de la racionalización en la conversación, sino del valor intrínseco de los pensamientos morales y mi éxito de vivir según estos. Para ACKERMAN la verdad moral es ni más ni menos que el nombre que le damos a las conclusiones a las que llegaríamos en una situación de diálogo ideal. En este sentido, hace una referencia directa al concepto de diálogo a la obra de HABERMAS.

3 ACKERMAN se refiere al artículo de DWORKIN (1978), para poder entender y completar los argumentos del poder constrictivo del diálogo en las sociedades liberales y analizar los nuevos signos de esta tipología de diálogo. ACKERMAN además cita en contraposición la teoría de HABERMAS (1976), como ejemplo de la tradición de los pensadores marxistas en este sentido. 
constrictiva provee de una clave más satisfactoria a la teoría liberal puesto que el concepto de diálogo en la obra de ACKERMAN no es el tipo de conversación idealizada de la que parte HABERMAS en su principio de democracia deliberativa. ACKERMAN afirma que en un diálogo liberal los ciudadanos no se sienten libres de introducir argumentos morales en el campo de la conversación, sino que intentan llegar a una victoria conversacional lejos de la situación ideal de diálogo (ACKERMAN, 1980, p. 19).

El autor considera que, a pesar de esta característica limitadora, es posible gobernar el mundo mediante el diálogo porque en las sociedades liberales modernas se realiza un tipo de depuración de la «constrictiveness» que llega a encontrar un diálogo neutro. Este tipo de diálogo neutro debe asegurar los siguientes aspectos fundamentales del Estado liberal, que permitirán un tipo de igualdad de oportunidades y un tipo de igualdad real en el punto de partida de los ciudadanos:

a) Uno de los requisitos básicos para poder hablar de diálogo en condiciones de neutralidad, es afirmar que no existe ningún tipo de diferencia en base a la genética. En este sentido podríamos ampliar el concepto a cualquier otro aspecto como raza, religión o lengua.

b) La educación, que tiene un papel central en la obra de ACKERMAN, debe proveer la base de los principios liberales a todos los ciudadanos del Estado.

c) La igualdad material se considera como necesidad de garantizar las condiciones para una igualdad de oportunidades. De aquí la importancia que ACKERMAN atorga a la justicia social ${ }^{4}$. Todo ciudadano empieza su vida adulta bajo las condiciones de la igualdad material.

d) Todo ciudadano puede cambiar libremente sus títulos iniciales mediante una red de transacción flexible.

e) Debe ser posible que las siguientes generaciones puedan aplicar el mismo sistema que las anteriores en condiciones de igualdad de oportunidades (B. ACKERMAN, 1980 , p. 28) ${ }^{5}$. Así pues, todo ciudadano, en el momento de su muerte, puede afirmar que ha cumplido sus obligaciones de confianza liberal si traspasa a la siguiente generación una estructura no menos liberal que la que recibió y disfrutó.

ACKERMAN se concentra en el desarrollo de los principios de justicia desde la conversación constreñida por el principio de neutralidad. Así, considera que los ciudadanos de una sociedad liberal deben estar sometidos a un diálogo neutro (THIGPEN y DowNING, 1983). El concepto de diálogo neutro se puede analizar desde dos ámbitos:

1. El principio de neutralidad como argumento filosófico. ACKERMAN considera que existe cierto paralelismo entre el rol de la conversación política en el Estado libe-

${ }^{4}$ En relación al principio de igualdad de oportunidades que ACKERMAN, HABERMAS y RAWLS establecen como elemento básico de sus teorías de la justicia se configura como uno de los principios básicos de la teoría liberal igualitaria. Esta igualdad de oportunidades es insensible a las posibles desigualdades derivadas de circunstancias de las que la gente no es responsable, como nacer en un determinado país, en cierta familia o tradición cultural. En este sentido destacamos la obra de GARGARELLA y OvEJERO, 2001, pp. 34 y ss. Coincidimos con el concepto de igualdad de oportunidades radical, establecido por G. A. COHEN, en GARGARELLA y OVEJERO, y que implica corregir las desigualdades debidas a diferencias de nacimiento o aquellas que aparecen como consecuencia de vivir bajo condiciones sociales desafortunadas y no escogidas. En este mismo sentido destaca la obra de ELSTER, 1999 con relación a un concepto de igualdad en un sentido más amplio.

5 El desarrollo de estos puntos son la base temática de la obra en su conjunto. Estos puntos son al mismo tiempo objetivos y las condiciones necesarias para poder construir un Estado liberal ideal. 
ral y el rol de la conversación filosófica en defensa del Estado liberal. La función de la conversación filosófica es hacer posible a una persona razonar según el principio de neutralidad sin declarar que el camino que ha elegido sea intrínsecamente mejor que cualquier otro camino del liberalismo (THIGPEN y DownING, 1983, p. 585). ACKERMAN no considera que la justificación del diálogo liberal necesite para su justificación respuestas a las principales controversias. La justificación última del liberalismo es haber encontrado en esta estrategia la localización de una red que permita la convergencia desde las diferentes direcciones (THIGPEN y DownING, 1983, p. 587).

2. El segundo enigma ante el que nos encontramos se refiere a los medios de socialización del principio de neutralidad en el Estado liberal. ACKERMAN considera que la socialización del principio de neutralidad se hará mediante la educación. El autor considera que la educación en un Estado liberal debe desarrollarse en dos etapas, la educación primaria, socialización realizada por los padres, que debe ayudar a controlar el comportamiento agresivo; y la educación secundaria, que debe ser llevada a cabo por la escuela y complementada por los padres. ACKERMAN considera que los niños necesitan coherencia cultural y la existencia de límites a la diversidad cultural que tiene que ser tolerada en una primera etapa de socialización (THIGPEN y DownING, 1983, p. 588).

Para ACKERMAN, la neutralidad es un principio básico, hasta el punto de ser considerado como punto de partida de su teoría liberal, que junto con los principios de consistencia y racionalidad, tienen que guiar el diálogo liberal. Aún así el autor no deja de reconocer el abuso absurdo que ha hecho el liberalismo de este concepto en la busca de una política libre de valores, lo cual comporta que su filosofía puede permanecer del todo superficial (ACKERMAN, 1983).

El autor asume de manera plena la definición de neutralidad establecida por R. DwORKIN por la cual una teoría liberal de neutralidad es aquella que analiza las cuestiones relacionadas con la justicia con total independencia de cualquiera idea de excelencia humana o de buena vida. Así, todas las discusiones sobre la legitimación de las relaciones de poderes tienen que ser sometidas al principio de neutralidad. El principio de neutralidad limita los tipos de argumentos que son aceptables para el debate liberal, pues ninguna razón no es buena si requiere de la fuerza para afirmar: a) que un argumento determinado es mejor que otro expresado por otro conciudadano, y $b$ ) que, a pesar de la concepción de bien, tal argumento es intrínsecamente superior a otro u otros argumentos de los conciudadanos.

El principio de neutralidad desarrolla en la teoría liberal de ACKERMAN el mismo rol que el velo de la ignorancia y la posición original realizan en la teoría de la justicia de J. RAWLS, a pesar de que ACKERMAN considera que el principio de neutralidad establece una condición hipotética superior a la establecida por RAWLS, puesto que este último manipula una mezcla entre ignorancia y conocimiento para favorecer conclusiones que justifiquen sus preferencias. ACKERMAN considera que su proposición es más realista que la de RAWLS y además permite a las personas saber cuáles son sus planes de vida (ACKERMAN, 1983).

En este sentido, J. S. FISHKIN sugiere una excelente comparación entre RAWLS y ACKERMAN (FISHKIN, 1983). Ambos buscan la solución para el problema de la justicia distributiva bajo condiciones ideales, ambos parten de un tipo de procedimiento 
de decisión imparcial. Para RAWLS este procedimiento es la posición original y para ACKERMAN es el principio del diálogo neutral bajo condiciones ideales de tecnologías perfectas de justicia. Los ciudadanos de la sociedad liberal deben aprender a dialogar con los otros ciudadanos de manera tal que les permita suprimir la condena a la moralidad del otro como mala o falsa, pues si no, la conversación pragmática quedaría vacía (ACKERMAN, 1989, p. 12).

Una de las principales diferencias a la hora de configurar el diálogo entre ambos autores se encuentra en cómo solucionar los desacuerdos entre los ciudadanos. HABERMAS, mediante su principio de democracia deliberativa, parece constituir un tipo de diálogo intemporal donde las personas deben llegar a decidir sobre cuestiones jurídicas o morales en base a argumentos racionales (HABERMAS, 1992). ACKERMAN, a diferencia de HABERMAS, afirma que en caso de desacuerdo entre los ciudadanos debemos aplicar el principio del «Conversational Restraint». Este concepto significa que, en caso de que los ciudadanos se encuentren en desacuerdo sobre las dimensiones de la verdad moral, no tenemos que buscar ningún valor común que ayude a superar el desacuerdo, sino que simplemente no tenemos que decir nada sobre este desacuerdo y sacar las ideas morales que nos dividen fuera de la agenda conversacional del Estado liberal (ACKERMAN, 1989, p. 16). Este tipo de autolimitación no tiene que suponer que perdamos la posibilidad de hablar los unos con los otros sobre nuestros más profundos desacuerdos morales en un contexto más privado. Restringiendo de este modo la conversación, hacemos un uso más pragmático del diálogo que se centrará en propósitos más productivos (ACKERMAN, 1989, p. 16).

ACKERMAN entiende el liberalismo como una manera de hablar sobre poder, como una cultura política de diálogo público basada en un determinado tipo de constricciones conversacionales (BENHABIB, 1993). Mediante este principio, considera que en la conversación liberal no se deberá decir nada que sea afirmativamente falso. En consecuencia, el diálogo servirá para supuestos de tipo pragmático y productivo. El principio, además, permite identificar las premisas normativas que todos los participantes encontrarán razonables (ACKERMAN, 1989, p. 17). El principio de constricción conversacional no se aplica a las cuestiones que los ciudadanos piden sino a las respuestas que legítimamente dan a otras cuestiones. ACKERMAN afirma que es posible concebir un mundo donde un determinado grupo de ciudadanos puedan solucionar los problemas relacionados con el poder enteramente basado en un concepto de diálogo neutro (ACKERMAN, 1980, p. 65). La cuestión que se plantea ACKERMAN es si los argumentos filosóficos trascendentales pueden ser adelantados para justificar uno u otro mediante el poder del habla (ACKERMAN, 1980, p. 8). Resulta empero que la mayor constricción conversacional al liberalismo es la neutralidad. ACKERMAN nos define la neutralidad como aquello donde «las normas que no son propuestas mediante la razón en un discurso de legitimación, pueden ser una buena razón para el poseedor del poder para afirmar que su concepción del bien es mejor que la afirmada por sus conciudadanos, o que su concepción del bien sin justificación es superior a la de uno o más de sus conciudadanos» (ACKERMAN, 1980, p. 11).

La división entre aspectos que se deben tratar en un tipo de esfera pública más abierta, y los aspectos que deben tratarse en esferas más privadas hace que BENHABIB afirme que la «Conversational Constraint» en ACKERMAN no sea neutra, puesto que 
supone una justificación implícita entre la esfera pública y la esfera privada, así como qué cuestiones se tienen que silenciar. En relación a esta consideración, coincidimos plenamente con S. BENHABIB cuando afirma que el principio de neutralidad no es de mucha utilidad a la hora de guiar nuestros pensamientos, puesto que se limita a afirmar que la Ley tiene que ser de tipo neutro (BENHABIB, 1993).

Como conclusión, podemos afirmar que el concepto de diálogo neutro tal y como lo establece ACKERMAN no es viable en el campo de la política o del Derecho puesto que no nos encontramos ante una ciencia exacta. En el campo del Derecho, cuando dos posiciones se oponen se usan todo tipo de argumentaciones posibles y por tanto hablar de un tipo de diálogo neutro no parece factible. Cualquier posición ecléctica que se pretenda adoptar establecerá en mayor o en menor medida algunos de los argumentos que son contradictorios. La imposibilidad práctica es todavía más grande cuando queremos legislar en base a las conclusiones que se puedan adoptar como consecuencia del diálogo neutro.

La positivización de la norma implica siempre una toma de decisión respecto aquello que se regula. A pesar de las condiciones que establece ACKERMAN para asegurar un diálogo neutro en condiciones determinadas no nos puede asegurar la generalización de su practicabilidad y efectividad reales, con todo sí que favorece su aplicabilidad a la teoría normativa de HABERMAS para mejorarla en el sentido de suponer un adelanto para poder llegar a conclusiones determinadas y acotadas en el tiempo por parte de los ciudadanos reunidos en la esfera pública. Podría otorgar, por tanto, cierto realismo al principio de Democracia Deliberativa aplicada a la legislación de las normas, sin suponer, como hemos dicho, la solución definitiva al problema en relación a la concepción del diálogo aplicada a la teoría habermasiana.

\section{MOMENTOS CONSTITUCIONALES Y MOMENTOS NORMALES}

B. ACKERMAN establece unas determinadas categorías analíticas que definen el sistema constitucional americano y sus transformaciones en base a su propia experiencia histórica. ACKERMAN afirma que el sistema democrático americano se ha visto muy influenciado por la teoría política europea a pesar de que los norteamericanos han creado un nuevo sistema constitucional que, a diferencia del modelo británico o alemán, distingue entre dos tipos de momentos políticos. Esta distinción es uno de los aspectos más importantes a destacar de su obra y además aplicable a la teoría de legitimación de normas jurídicas de HABERMAS y, en especial, al proceso de codificación de las normas. Según el tipo de momento político ante el que nos encontremos, el diálogo de los ciudadanos será de un modo u otro con su correspondiente trascendencia jurídica.

Los momentos constitucionales se dan raramente, cuando «We the People» hablamos de manera directa. En estos periodos, el pueblo habla mediante vías extraconstitucionales, y son momentos en que los ciudadanos quieren realizar cambios en la Constitución (NEBELSKY, 1994). Estos momentos están caracterizados por distintas circunstancias; primero, por el hecho de que un número extraordinario de ciudadanos está convencido de la seriedad del asunto que se discute, seriedad mucho mayor que la seriedad que se otorga a las decisiones políticas normales; segundo, por el hecho 
de que todos los ciudadanos han tenido la posibilidad de organizarse para expresar su forma de ver el problema que se discute; y, tercero, por el hecho de que existe una mayoría partidaria de una determinada forma de solucionar el problema en cuestión (ACKERMAN, 1991, p. 16).

En los momentos constitucionales la sociedad se moviliza para debatir asuntos fundamentales. En estos momentos existe un grado de reflexión y de deliberación popular inusual por parte de la ciudadanía que pretende realizar una transformación de tipo constitucional. Partiendo del análisis de la historia constitucional de los Estados Unidos, ACKERMAN nos explica de manera completa cómo los ciudadanos actúan, se movilizan y dialogan en los momentos constitucionales que transforman la constitución americana. Los tres momentos que ACKERMAN considera como constitucionales son el establecimiento del marco básico durante la década de 1780, las reformas de la «Reconstruction» realizada por los Republicanos durante la década de 1860, y el denominado «New Deal» de los Demócratas a partir de 1930. Estos momentos constitucionales provocaron una reinterpretación de la Constitución de los Estados Unidos mediante resoluciones del Tribunal Supremo y enmiendas constitucionales (ACKERMAN, 1993). En este tipo de momentos, los ciudadanos se encuentran en un estado de exaltación enorme y se genera una necesidad de actuación directa que suele contradecir los propósitos de actuación del gobierno de turno. Esta contradicción entre la decisión del gobierno y la voluntad popular es lo que genera la exaltación.

Los acuerdos a los cuales llega la ciudadanía en los momentos constitucionales bajo circunstancias excepcionales se encuentran, según ACKERMAN, en el mismo peldaño jerárquico que las reformas formales de la Constitución, porque, en definitiva, lo que otorga valor a las normas constitucionales no tiene que ver con la calidad personal de sus autores o el momento particular en que fueron dictadas, sino por el extraordinario nivel de acuerdo ciudadano que las apoya.

ACKERMAN, a diferencia de otros autores, considera que los momentos constitucionales se producen exclusivamente en momentos convulsivos. No debemos olvidar que, en la formulación original de ACKERMAN, los momentos constitucionales son breves y extraños (sólo tres en doscientos años de historia). BALKIN y LEVINSON no consideran como conditio sine qua non este estado convulsivo, sino que los momentos constitucionales se producen mediante un proceso que denominan «partisan entrenchment» por el cual un partido (Pueblo, Presidente del Gobierno, Partidos políticos o Poder judicial) coge el control, normalmente de manera repentina, pero en ocasiones gradualmente, de las tres ramas del gobierno (BALKIN y LEVISON, 2000, III). En este sentido debemos aclarar que para ACKERMAN, el punto fundamental de la separación de poderes en los Estados Unidos es que ninguna rama del gobierno no puede reclamar por sí sola hablar en nombre del pueblo (NEDELSKY, 1994).

$\mathrm{Al}$ respecto, TUSHNET pone determinadas sentencias judiciales producidas durante el «New Deal» como ejemplo de momentos constitucionales sin convulsión asociada (TUSHNET, 2003) ${ }^{6}$. En este mismo sentido NEBELSKY afirma que las transformaciones

\footnotetext{
${ }^{6}$ En especial destacamos el capítulo segundo del libro: «The Supreme Court and the New Constitutional Order», donde se analizan un conjunto de decisiones judiciales que podían considerarse como momentos constitucionales provocados por el poder judicial sin que la ciudadanía de los Estados Unidos se haya encon-
} 
dramáticas que producen los momentos constitucionales no tienen en cuenta suficientemente los importantes cambios que los jueces han introducido en la significación de los derechos constitucionales. La autora continúa desarrollando en su trabajo casos determinados, como por ejemplo Grinswald and Brown o Plessy vs Fergusson, donde los jueces han redefinido derechos fundamentales como la igualdad. Según NEBELSKY, el poder judicial con esta «constitutional adjucation» establece cambios equivalentes a los momentos constitucionales (NEDELSKY, 1994, p. 515). Sobre la necesidad de que se produzca un momento convulsivo en la sociedad para tener un momento constitucional, no podemos olvidar que si bien la Constitución es una norma de concordia, las normas constitucionales son esencialmente leyes de discordia (ZAGREBELSKY, 2005, p. 115). ACKERMAN establece un tipo de criterio formal que determinará si nos encontramos ante un momento constitucional o no, con independencia del estado de convulsión de la ciudadanía. Tal y como afirma TuSHET, las obligaciones de fidelidad a la Constitución se acentúan en los momentos constitucionales y los ciudadanos requieren claridad sobre en qué precisas ocasiones se encuentran ante un momento constitucional del que surgirá la obligación de fidelidad (ZAGREBELSKY, 2005, p. 115). Los momentos políticos normales se dan mediante los representantes, mientras que los constitucionales por la ciudadanía misma; los momentos normales no son una particularidad reflexiva, los constitucionales sí que lo son. Además, HERZOG añade un tercer criterio consistente en que los momentos normales están relacionados con la persecución plural de los grupos de interés, mientras que los constitucionales están vinculados con principios y con el bien común (HERZOG, 1994). Los momentos constitucionales también se caracterizan por su rol al alterar el marco por el cual los momentos ordinarios se desarrollan, es decir, que el momento constitucional no sólo se diferencia por sí mismo del momento normal que le ha precedido y que le seguirá, sino que además, debe asegurar que las dos fases del momento ordinario, antes y después, son totalmente diferentes una de otra. El momento constitucional está marcado, por tanto, por la discontinuidad y la transformación (WALKER, 1993). Si aceptamos este análisis, estamos introduciendo además un criterio que nos ayudará a determinar cuándo nos encontramos ante un momento constitucional y cuándo no. Por tanto, podemos caracterizar los momentos constitucionales como altamente infrecuentes, se limitan a momentos abruptos de la política, tienen una repercusión constitucional considerable y tienen efectos duraderos, a pesar de que el momento constitucional no es perdurable por sí mismo. El dato más importante a efectos del presente trabajo es que la ciudadanía pretenda realizar una transformación de tipo constitucional de manera directa.

En contraposición de los momentos constitucionales encontramos los denominados momentos normales. Dentro de estos momentos normales incluimos las decisiones que toma a diario el gobierno. En estos momentos no hay debate ni movilización popular. El electorado confía al gobierno la gestión de los negocios jurídicos, y el gobierno, legitimado por este mandato, adopta las decisiones que cree más convenientes (ACKERMAN, 1991, p. 16). En los momentos normales una población «desligada» permite a los grupos de interés democráticamente escogidos tomar decisiones políticas.

trado en un momento convulsivo determinado. El autor considera que el nuevo orden constitucional ha sido consolidado con las elecciones presidenciales de los Estados Unidos del año 1994 que provocaron la mayoría republicana. 
Un elemento realmente importante de los actos políticos normales es el hecho de que en estos momentos políticos los representantes toman decisiones en nombre de los ciudadanos. La implicación cívica en la toma de decisiones es baja. A pesar de que parecería que el estudio de los momentos normales es menos interesante, ACKERMAN afirma, correctamente, que es necesario ser muy respetuosos con lo que sucede en los momentos normales, es decir, con la decisión del pueblo de retirarse de la política, delegando la toma de decisiones en sus representantes (GARGARELlA, 2005, p. 3). No en vano, «el pueblo» puede decidir libremente retirarse de la política y delegar en sus representantes, que por otro lado, pueden ser sustituidos mediante los medios democráticos oportunos.

Los momentos normales, por tanto, son igual de importantes que los constitucionales por la estabilidad y continuidad necesaria a cualquier ordenamiento jurídico. Tal y como hemos adelantado anteriormente, la finalidad de los momentos constitucionales es afectar de manera directa a un momento normal determinado para producir otro de diferente. ACKERMAN destaca de los momentos normales que la carencia de debate y de participación popular no vicia necesariamente la legitimidad de las decisiones tomadas por el gobierno si determinadas condiciones institucionales son satisfechas. Estas condiciones son, por ejemplo, que los representantes del pueblo puedan ser responsables de sus decisiones; segundo, que la estructura institucional obligue los representantes del pueblo a tomar decisiones en base a una visión amplia del interés público; tercero, que exista una legislación que impida que grupos de intereses condicionen el gobierno para obtener ventajas desleales.

La teoría y experiencia de los momentos constitucionales tiene que ser estudiada con detenimiento si queremos poner en práctica la teoría habermasiana de democracia deliberativa, puesto que los ciudadanos tienen acceso directo a la promulgación de las normas fundamentales de su Estado sin intermediarios. El estudio de cómo «el pueblo» ha actuado de manera directa en estos momentos tiene que partir de la diferenciación del tipo de normas que se codificará; de ahí la importancia de la distinción entre momentos normales y momentos constitucionales, y las características que se dan en ambos momentos. ACKERMAN analiza cómo la ciudadanía obliga a sus ramas representativas a actuar o legislar de una determinada manera, a pesar de que este proceder sea contrario a la constitución del país. En los momentos constitucionales, los ciudadanos, por tanto, usan los medios habituales de codificación, es decir, mediante los órganos representativos: Parlamento, Presidente, Poder judicial, para cambiar el contenido de los preceptos constitucionales. En consecuencia, «el pueblo» no actúa en un ágora imaginaria, de manera directa y mediante un diálogo pacífico y neutro, más bien al contrario.

El esquema de ACKERMAN, a diferencia de la teoría de HABERMAS, se basa en una experiencia histórica real que debe tenerse en cuenta en el momento de aplicar el principio de democracia deliberativa aplicada al Derecho. Otra novedad introducida por ACKERMAN, y que podría mejorar los propósitos empíricos de la teoría de HABERMAS, es que el primero acota el diálogo en el tiempo. HABERMAS considera que las personas pueden mantener el diálogo indefinidamente hasta que la decisión se tome por unanimidad entre todos los posibles afectados por la norma. ACKERMAN limita el marco del diálogo en momentos determinados acotados en el tiempo. Incluso en los momentos 
de mucha intensidad (constitucionales) el movimiento ciudadano es determinado en el tiempo. Siguiendo los criterios de diferenciación que usa ACKERMAN entre momentos constitucionales y momentos normales, podemos observar cierta analogía entre los momentos constituyentes y los momentos no constituyentes.

ZAGREBELSKY, siguiendo la línea de ACKERMAN, afirma que el verdadero momento constitucional es sólo el momento inicial, es decir, el momento constituyente. Afirma que es mucho más sencillo crear una constitución de la nada que modificar el contenido sustancial de una constitución ya existente. Estas reformas complicadas pueden ser causa de la convulsión que se genera en los momentos constitucionales no constituyentes. El «momento constitucional» es por definición el momento de cooperación general, tiene unas características completamente excepcionales en la vida política de un pueblo, puesto que una Constitución no se puede crear y recrear a placer (ZAGREBELSKY, 2005, p. 116) ${ }^{7}$. En los momentos constituyentes, las voluntades políticas de los sujetos políticos se coordinan para la consecución de un objetivo común; dictar unos principios que se sitúan por encima de los intereses particulares de cada uno de ellos, posibilitando la convivencia de todo el mundo. Un ejemplo actual de momento constituyente lo encontramos en la refundación del Estado de Bolivia, cuando un tipo de momento constitucional generado por la población originaria del país andino pugna por una nueva constitución política que reconozca estos pueblos y sus culturas en un plano de igualdad.

Otro elemento en común entre los momentos constitucionales y los momentos constituyentes es que ambos tipos de acontecimientos se producen en forma de «événement» en sentido foucaultiano. Es decir, se produce una discontinuidad al discurso jurídico, si bien, en los momentos constituyentes, esta discontinuidad proviene de una revolución, tal y como afirma $\mathrm{H}$. ARENDT, y los momentos constitucionales suceden en un estado de convulsión, tal y como afirma ACKERMAN (ARENDT, 2006) ${ }^{8}$.

Cuando HABERMAS configura su principio de democracia deliberativa no diferencia entre ningún tipo de momento. En consecuencia, el pueblo no otorga ningún tipo de mandato a sus representantes para gestionar los negocios políticos o jurídicos, puesto que los ciudadanos, mediante el Principio de Democracia Deliberativa, deciden sobre los aspectos políticos o jurídicos. Entendemos que la teoría y experiencia de los momentos constitucionales y normales debe ser estudiada con detenimiento si queremos poner en práctica la teoría habermasiana de democracia deliberativa, puesto que los ciudadanos tienen acceso directo a la promulgación de las normas fundamentales de su Estado sin intermediarios. El estudio de cómo «el pueblo» ha actuado de manera directa en estos momentos tiene que partir de la diferenciación del tipo de normas que se codificará, y de ahí la importancia de la distinción entre momentos normales y momentos constitucionales, y sus características. A partir de esta diferenciación podemos

7 Contra esta afirmación entendemos que las constituciones o bien las normas que desarrollan el contenido constitucional, como normas jurídicas que son, han de poder reformarse para poder actualizar el marco que regulan y no quedar obsoletas.

8 En este punto, vid. Monserrat y Abad, 2006, donde destacamos las influencias del concepto de revolución de H. ARENDT sobre la dialéctica constitucional y la diferenciación entre los momentos constitucionales y normales. En relación al concepto d'«événement» y la teoría de la discontinuidad destacamos las obras de M. Foucault, Dits et écrits II, Paris, Gallimard, 2001, pp. 140-149, y Les Mots et les Choses, Paris, Gallimard, 1990. 
entender que el diálogo entre los ciudadanos variará según qué tipo de norma jurídica, hecho a codificar o estado de convulsión de la población determinada, y que, por tanto, no se puede establecer un sometimiento general a principios del discurso sin tener en consideración estos elementos.

\section{DEMOCRACIA DUALISTA}

La diferenciación entre ambos tipos de momentos políticos expuesta en el apartado anterior se enmarca dentro del análisis del concepto de democracia dualista. La democracia dualista consiste en diferenciar entre las decisiones tomadas por el pueblo mismo y las decisiones tomadas por el gobierno. Por tanto, el concepto de democracia dualista descansa sobre la diferencia entre momentos constitucionales y momentos normales antes introducida. La teoría dualista de la democracia se contrapone a la teoría monista. El monismo sigue la tarea de la teorización constitucional de reconciliar la autoridad de los poderes públicos no electos, en especial en el sistema de división de poderes (poder judicial). La solución monística es una presunción de validez legislativa (POSNER, 1992). La democracia monista consiste básicamente en la idea que la democracia requiere que gobiernen aquellos representantes escogidos por el pueblo, a quienes se ha otorgado el pleno poder de dictar leyes, en tanto en cuanto esta elección ha sido libre. La consecuencia institucional más importante del monismo es que durante el periodo que va de elección a elección cualquier control que intente recortar las atribuciones del gobierno es visto como un acto antidemocrático (ACKERMAN y ROSENKRANTZ, 1991, p. 19). La idea del monismo es muy simple y atractiva para cualquiera que crea en la democracia y la representación popular y, en consecuencia, en el carácter antimayoritario de las decisiones judiciales. Para el monismo no hay más autoridad democrática que un Parlamento democráticamente escogido. Para esta teoría, la distinción básica del dualismo que diferencia entre las decisiones del pueblo y las decisiones de los representantes del pueblo no tiene sentido. El monismo no establece diferencias en cuanto a la legitimidad y validez de ambos tipos de decisiones (ROSENKRAFT y ACKERMAN, 1991, p. 19).

La introducción y el desarrollo de la democracia dualista es uno de los aspectos fundamentales tratados a lo largo de la obra de B. ACKERMAN. El autor analiza qué tipo de actos y decisiones políticas pueden ser considerados como dualistas y cuando se producen este tipo de actos. Tal y como hemos mencionado anteriormente, los momentos constitucionales se dan raramente, cuando el pueblo habla directamente; por otra parte encontramos los momentos normales, cuando sólo habla el gobierno.

ACKERMAN nos recuerda que la democracia ha sido pensada para los ciudadanos y el sistema tiene que poder permitir la participación directa de los ciudadanos. A lo largo de la historia, especialmente en los últimos dos siglos, la sociedad ha recuperado el término «democracia» para transformarlo en algo banal en una reconceptualización que ha desplazado la imagen de la polis griega como modelo de gobierno popular, centro del pensamiento y práctica democrática (ACKERMAN, 1993, p. 295; cfr. MCILWAN, 1947). Ejerciendo su derecho al voto, los ciudadanos privados no consideran que sus votos puedan significar un cambio fundamental en los principios. Para que tal posibili- 
dad de cambio pueda llegar a ser apreciada, se debe informar al ciudadano en general y se tiene que dar la opción de deliberar sobre las proposiciones transformativas. En caso contrario, los políticos escogidos presumen que han recibido el consentimiento del pueblo para mantener su status y sus concepciones sobre los principios (ACKERMAN, 1993, p. 307). En este sentido, la falta de expectativas y posibilidades de cambios reales son causas de la baja participación de los ciudadanos en las elecciones democráticas. Esta falta de participación es un factor que tiene que ser resuelto si se quieren considerar como legítimos determinados resultados electorales.

ACKERMAN considera que mediante el sistema de democracia dualista se puede ofrecer un tipo de alternativa a esta involución democrática. El sistema de democracia dualista pretende captar la iniciativa privada de los ciudadanos en las sociedades liberales y canalizarlo a la vida pública. Así, uno de los elementos fundamentales de la teoría dualista de ACKERMAN es el concepto de ciudadano activo, es decir, los ciudadanos que actúan de manera participativa en la vida pública de la ciudad. No podemos obviar el hecho de que los conceptos de democracia dualista y de momento constitucional, en cuanto que dependen de la actuación directa de los ciudadanos, descansan en el concepto de «ciudadano activo». En este sentido, ACKERMAN considera que muchos de los ciudadanos de los Estados Unidos son ciudadanos privados, no ciudadanos públicos o ciudadanos privados perfectos (GREENAWALT, 1994).

Los «ciudadanos públicos» son aquellos ciudadanos devotos del bien público cuyas formas primarias de vida son políticas. Los «ciudadanos privados perfectos» persiguen exclusivamente objetivos no políticos, y usan el proceso político para conseguir estos objetivos. Los ciudadanos privados normalmente actúan de manera privada, votando y cumpliendo algunas responsabilidades mínimas como ciudadanos, pero atendiendo de manera primaria aspectos personales y tratando el proceso político como un modo de conseguir intereses individuales y de grupo. En ocasiones, los ciudadanos privados actúan dándole mucha más atención al bien público, como por ejemplo durante los años sesenta cuando muchos ciudadanos privados actuaron como ciudadanos en la defensa de los derechos civiles y oponiéndose a la guerra de Vietnam (GREENAWALT, 1994). Según este diseño, la Constitución de los Estados Unidos de América funciona de manera ingenua en relación a los ciudadanos privados (ACKERMAN, 1993, p. 240). La mayoría de las instituciones políticas están establecidas por los periodos ordinarios. En estos periodos, donde la mayoría de los ciudadanos no están concentrados en el bien público, las acciones legislativas y ejecutivas no reflejan al Pueblo en su capacidad colectiva. La representación disminuye las posibilidades que una simple facción de ciudadanos gane el control o ayude a la producción legislativa. Los cambios constitucionales drásticos son posibles cuando el Pueblo, durante los momentos constitucionales, demuestra su fuerte apoyo duradero a los cambios constitucionales (GREENAWALT, 1994).

El concepto de democracia dualista se configura en base a la concepción de ciudadano privado, que se mantiene entre la privacidad cívica y el espíritu público comprensivo que la teoría de ACKERMAN urge (GALTSON y GALSTON, 1994). ACKERMAN considera equivocado el silogismo de partida de muchos ciudadanos privados que afirman: «lo que es bueno para mí es bueno para mi país». ACKERMAN considera que la segunda parte de esta afirmación es la realmente importante, es decir, en caso de que aparezca un tipo de conflicto de intereses prevalecerá lo que es bueno para el 
país. El autor nos detalla cómo saber cuándo divergen los intereses públicos de los privados y acaba afirmando de manera correcta que un ciudadano no puede considerarse como ciudadano privado si no puede deliberar sobre los bienes públicos (ACKERMAN, 1993, p. 297).

ACKERMAN considera que el dualismo se dará tarde o temprano en el sistema democrático; además debe existir un tipo de acuerdo entre la libertad personal y la historia constitucional (revolucionaria) de los Estados Unidos de América. El trato se basa en dos argumentos o presuposiciones básicas. El primero surge de la misma concepción de ciudadano privado: todos los ciudadanos privados en cualquier momento pasan por un momento de voluntad de participación en la vida pública. Los ciudadanos no se encuentran siempre de manera pasiva ante lo que pasa en la sociedad. Esta pasividad varía y es en este periodo donde el ciudadano ha sido asesorado de la necesidad de preservar el lenguaje y la práctica dualista que harán posible el ejercicio de la deliberación de los ciudadanos activos.

El segundo argumento no trata tanto de cómo los ciudadanos actuaran en un momento determinado. ACKERMAN valora los elementos que distraen los ciudadanos en relación al bien público de la nación como un todo. Estos aspectos son su trabajo, su familia, sus amigos, su religión, su cultura. ACKERMAN afirma que esta diversidad de experiencias de vida es uno de los éxitos fundamentales de América y no se podría haber conseguido si todo el mundo tuviera la ciudadanía nacional como prioridad (ACKERMAN, 1993, p. 299). Con esta afirmación, ACKERMAN nos vuelve a demostrar la necesidad de momentos de pasividad en la ciudadanía, es decir, vuelve a destacar la necesidad de los momentos normales en el sistema dualista. ACKERMAN considera que la vinculación de las personas en la vida pública tiene que ser entendida como un precio que se paga por la libertad sin interferencias del poder.

Una de las críticas que ha recibido la concepción de ACKERMAN es en relación a la falta de conciencia del Pueblo a la hora de emprender una reforma constitucional. Es decir, a pesar de producirse una reforma constitucional, acontecida en un momento constitucional, el pueblo no tiene conciencia directa de las reformas de principios constitucionales que provoca su actuación (POSNER, 1992). Esta conciencia es trascendente desde una perspectiva histórica en el ejercicio de la soberanía popular en momentos revolucionarios, y cómo estos afectan a la hora de determinar la fundamentación de los derechos.

ACKERMAN completa el concepto de democracia dualista basada en una nueva carta de derechos, es decir, en una igualdad de oportunidades efectiva que afecte las escuelas, los trabajos y los lugares públicos (ACKERMAN, 1993, p. 319). La idea de democracia dualista es compartida por RAWLS y tiene efectos parecidos a los buscados por HABERMAS mediante su principio de democracia deliberativa.

En este sentido, GARGARELLA afirma que «RAWLS muestra su propia teoría en confluencia con la visión desarrollada por B. ACKERMAN en sus estudios sobre el constitucionalismo. Como este, RAWLS considera que la democracia es "dualista", es decir, una democracia donde se diferencia el poder constituyente del poder ordinario, tanto como la ley superior del pueblo, de la ley ordinaria de los cuerpos legislativos» (GARGARELLA, 2005, p. 3). 
GARGARELLA continúa afirmando que la teoría de democracia dualista de ACKERMAN tiene determinados inconvenientes y afirma que «la visión dualista falló por no reconocer los infinitos matices existentes entre decisiones aprobadas en "momentos constitucionales" y decisiones aprobadas en "momentos corrientes". Si alguien quisiera tomar seriamente la lógica que la mencionada proposición ofrece, entonces, haría bien al distinguir entre decisiones democráticas de distinto tipo de importancia» (GARGARELLA, 2005, p. 3). En este mismo sentido, MORTON afirma que «tanto conceptualmente como sociológicamente, los modelos de democracia deliberativa y dualista se centran en el proceso de interdependencia recursiva y hermenéutica que existe entre la política democrática y el poder constituyente» (HoRWITZ, 1993, p. 107).

Uno de los otros temas fundamentales de la división entre un sistema democrático monista y un sistema dualista es cómo afectan cada una de las teorías a la tensión existente entre democracia y revisión judicial constitucional (MiCHELMAN, 1999). Resulta evidente que la tarea del Tribunal Supremo y toda la cuestión del control constitucional diferirá enormemente según se crea que el monismo o el dualismo sean la mejor concepción de la democracia. Para el monismo, el control constitucional es básicamente un acto antimayoritario, y consecuentemente la justificación del control del constitucional por parte del Tribunal Supremo, si se da, vendrá legitimada por el especial rol del Tribunal Supremo o de la naturaleza procedimental respecto al proceso democrático de los derechos involucrados. De manera contraria, el dualismo ve el Tribunal Supremo y el control de constitucionalidad como una institución de preservación de una sociedad democrática bien ordenada que sirve para proteger la democracia salvando las decisiones de una ciudadanía movilizada contra las decisiones de los representantes que no han obtenido la clase de apoyo requerida para obtener la máxima legitimidad (ROSENKRAFT y ACKERMAN, 1991, p. 19).

Este es, sin ningún tipo de duda, uno de los elementos más problemáticos de la construcción de la teoría dualista, que otorga la potestad de reforma constitucional a los ciudadanos mediante el poder judicial, sobre todo teniendo en cuenta que el poder judicial no acepta que la Constitución sea enmendada por vías diferentes a las establecidas de la forma clásica (GREENAWALT, 1994).

La concepción dualista no afirma que cualquier decisión del Tribunal Supremo pueda ser justificada en términos preservacionistas, es decir, la idea de que el Tribunal Supremo debe intervenir para preservar las decisiones del Pueblo adoptadas en momentos constitucionales. Para determinar cuándo el Tribunal Supremo está legitimado para preservar las decisiones del Pueblo debemos tratar el problema preliminar y determinar cuáles son las iniciativas que han obtenido la más alta legitimidad para ser consagradas por una ciudadanía movilizada y activa (ROSENKRAFT y ACKERMAN, 1991, p. 19). En este punto volvemos a destacar la diferencia entre momentos constitucionales y normales desarrollada en el apartado anterior y, en especial, los criterios de diferenciación entre ambos tipos de momentos. Obviamente, esta distinción es intranscendente para los partidarios de la concepción monista de la democracia y de la adjudicación constitucional. Desde la concepción monista se cree que el Tribunal está realizando una actividad presuntamente antidemocrática cuando este órgano jurisdiccional invalida una ley aprobada por mayoría parlamentaria democrática. 
ACKERMAN es consciente que su formulación incluyendo las enmiendas no formales de la Constitución no se encuentra entre las teorías jurídicas habituales. Su conexión entre la justificación judicial y una teoría constitucional más amplia es una de las cuestiones cruciales de su obra (ROSENKRAFT y ACKERMAN, 1991, p. 19). Las teorías monistas se centran en una idea de justicia que tiene que servir para cualquier tipo de disputas. Los dualistas, siguiendo a Aristóteles, afirman que la justicia se presenta de dos maneras: correctiva y distributiva. ACKERMAN intenta incorporar elementos de ambas escuelas a una concepción de justicia más compleja (ACKERMAN, 1997).

Para posibilitar este acceso, ACKERMAN usa el concepto de «Corrective Justice». Este concepto aplicado a los individuos hace que las personas abandonen sus experiencias propias de vida, centrándose a comprender el problema de la justicia en términos que den sentido sin la afectación de temporalidades por las cuales la vida se ve afectada día a día. El principio de justicia correctiva se centra de manera implacable en las personas que interrumpen experiencias particulares en momentos particulares. La búsqueda de un actor responsable empieza con la interrupción de una experiencia de vida particular, que no debería haber acabado en aquel momento determinado (ACKERMAN, 1997). Este último momento determinado sigue siendo el punto de vista decisivo para valorar el punto de experiencia de partida y, por tanto, donde deberá actuar el principio de justicia correctiva.

La teoría de justicia ecléctica que afecta a los ciudadanos es un tipo de justicia relacional, es decir, basada en las relaciones entre las personas. En este sentido, las experiencias particulares de las personas tienen una trascendencia limitada. Aquello realmente importante es la valoración que damos a estas experiencias y cómo afectan a los principios de la justicia. La idea fundamental que expresa ACKERMAN es que la justicia requiere el respeto mutuo y la posibilidad que deben tener otros de expresar sus propias historias sobre nuestros relatos. Además, el principio de justicia relacional requiere nuestro reconocimiento a las contribuciones realizadas por los otros a los campos en que participan. En esta tarea, ACKERMAN pretende establecer un contexto determinado mediante el cual los ciudadanos puedan seguir la realización de la justicia en una sociedad democrática (ACKERMAN, 1997).

ACKERMAN afirma que a pesar de la inmensa diversidad existente entre los ciudadanos en la sociedad moderna, los ciudadanos y la existencia de los tres horizontes temporales de cada persona (experiencia de vida, relaciones significativas e historia vital), se puede llegar a una teoría de justicia estableciendo un factor correctivo y conectando este aspecto metafísico con el tema en cuestión planteado. Tal y como hemos adelantado anteriormente, en relación con la democracia dualista, existen grandes similitudes entre la teoría de ACKERMAN y la teoría de HABERMAS. En este sentido, ambos autores proponen un nuevo examen de las constituciones contemporáneas y su sistema constituyente centrándose en diferentes tipos de justificación democrática; ambos autores ofrecen un modelo procedimental de legitimación democrática y, finalmente, en ambas teorías la legitimación es otorgada mediante la deliberación pública.

La diferencia entre ambos autores radica en que ACKERMAN propone una estricta separación entre la experiencia constitucional y los aspectos políticos de los ciudadanos. HABERMAS, en contraste, establece una teoría más dinámica y abierta a las deli- 
beraciones públicas de los ciudadanos. Según VARgova, la teoría de AcKerman que diferencia entre momentos constitucionales y normales permite dividir estrictamente entre diferentes esferas públicas (política, Derecho y la vida social de la democracia liberal), construyendo una jerarquía normativa, política y una sociedad dividida socialmente, mientras que HABERMAS establece un concepto de dualismo dinámico, distinguiendo entre varios tipos de discursos formales e informales de deliberación pública, acentuando el rol democratizador de la esfera pública y la sociedad civil para la legitimación de la deliberación constitucional (VARGOVA, 2005, p. 365).

En este sentido, VARGOVA aplica la teoría de las esferas públicas de HABERMAS a la obra de ACKERMAN aplicando el factor corrector de FRASER. VARGOVA afirma que la teoría dualista de ACKERMAN tiene el déficit importante de otorgar únicamente a momentos extraños el ejercicio de deliberación de la ciudadanía democrática. Según la autora, ACKERMAN infravalora la actividad pública de los ciudadanos y los esfuerzos diarios que llevan a cabo mediante los procedimientos ordinarios. En el sistema dualista de ACKERMAN, los ciudadanos son tratados bajo un tipo de estereotipo político por el que en los momentos normales, la ciudadanía o no quiere o no puede participar en las discusiones públicas y que sus juicios sean considerados. ACKERMAN, por tanto, despreciaría el día a día de los ciudadanos y sus esfuerzos de autorregulación democrática (VARGOVA, 2005, p. 365). No coincidimos con el diagnóstico hecho por VARGOVA.

Con la formulación original de la teoría dualista, ACKERMAN hace un diagnóstico de cómo ha reaccionado el pueblo a lo largo de los doscientos años de historia a los Estados Unidos. Separa claramente los momentos constitucionales en que la ciudadanía quiere cambiar principios o normas de rango constitucional, de los momentos normales en que los ciudadanos, mediante las vías establecidas, pueden cambiar normas de rango inferior. ACKERMAN, además, en todo momento denota la importancia de los momentos normales como hemos mostrado en el apartado anterior del presente trabajo. Entendemos que la ciudadanía puede participar en la vida política del país, sin que necesariamente tenga que producirse un momento de tipo constitucional. Sólo es necesario recordar que los momentos constitucionales suceden y preceden a los momentos normales, que se verán afectados por el contenido del momento constitucional, es decir, por la voluntad del pueblo de manera directa. Una crítica diferente es que se hayan dado más momentos constitucionales, durante los últimos dos siglos de historia de los Estados Unidos, de los que destaca ACKERMAN, o que determinadas resoluciones judiciales han provocado el mismo efecto que los momentos constitucionales sin que se haya producido el estado de convulsión pública que predica ACKERMAN.

VARGOVA defiende el concepto de esfera pública y el procedimiento de deliberación democrática de HABERMAS por considerarlo más plural, dinámico e inclusivo que la arena que establece ACKERMAN, y, en consecuencia, el único sistema que no desprecia a los ciudadanos resulta ser un tipo de monismo a la inversa, es decir, los ciudadanos actúan de manera directa en todos los casos. Con independencia de estar de acuerdo o no con la formulación, no es este el objeto de la teoría de ACKERMAN.

Coincidimos en este sentido, a pesar de que nuestra propuesta se quiere ecléctica entre ambos autores, pues, partiendo de elementos en común, pretendemos proponer una teoría de compatibilidad y no establecer un tipo de jerarquía entre ambas teorías, 
además de proponer, desde nuestra perspectiva filosófica, el estudio de la afectación al Derecho distinguida de la esfera política?

\section{CONCLUSIÓN}

Hemos definido los aspectos principales de la obra de B. ACKERMAN relacionados con la definición, exposición de las características y críticas de los conceptos de diálogo neutro y neutralidad que entendemos que podrían mejorar y dar más virtualidad al Principio del Discurso de HABERMAS aplicados al Derecho. Consideramos que también la diferenciación entre momentos constitucionales y normales, en base a la experiencia constitucional de los Estados Unidos de América, debería ser tenida en cuenta para establecer un marco para el discurso jurídico y su aplicación en la legislación de normas jurídicas legítimas, especialmente si se trata de normas fundamentales.

Con estas consideraciones tratamos de aclarar que no se puede pretender establecer un Principio de Discurso que afecte a contenidos jurídicos y maneras de legitimación de las normas sin tener en cuenta determinadas prevenciones básicas. También hemos tratado aspectos como el necesario respecto a las minorías y el tratamiento que se tiene que dar a las segundas generaciones. Estos aspectos necesarios tampoco han sido trabajados por HABERMAS a la hora de establecer un principio democrático deliberativo intemporal y universal.

Entendemos que, aplicando estas correcciones, el proyecto de HABERMAS ganaría en calidad democrática e incluso en universalidad. En este sentido, no podemos olvidar el hecho de que se han puesto en práctica muchas experiencias de democracia deliberativa aplicadas al Derecho a lo largo de la historia. De estas experiencias, los autores deberían extraer conclusiones e información para intentar llevar a cabo sus propuestas de manera mejorada y renovada (HERMAN, 1999, p. 179) ${ }^{10}$.

Entendemos que compaginando estos aspectos de la obra de ACKERMAN a la teoría de legitimación de normas jurídicas propuesta por HABERMAS conseguiremos un proyecto de democracia radical más coherente y más eficaz. Con estas consideraciones y correcciones consideramos que la obra de HABERMAS iría más allá de una intuición especial de un filósofo moral y de una teoría especulativa deontológico-universal para encaminarse más efectivamente a ayudar a tomar las decisiones adecuadas de carácter

${ }^{9}$ En este sentido consideramos necesario destacar la diferencia existente entre el lenguaje jurídico y el lenguaje político, así como la diferencia entre la ciencia política y la ciencia jurídica. HABERMAS parece confundir ambos campos en su obra. Esta apreciación también es compartida por ACKERMAN, tal y como lo corroboró una de las reuniones que mantuvimos en su despacho de Yale en agosto de 2005. La confusión entre los dos campos tiene repercusiones importantes en la teoría de HABERMAS, ya que aplica parámetros políticos al Derecho y a la inversa.

${ }^{10}$ El Tribunal del Pueblo es una traducción de la palabra griega dikasterion que por sí misma significa Tribunal de Ley, pero fue utilizada en Atenas por los grandes jurados que fueron la característica distintiva de la democracia. En las ciudades estado democráticas, la dikasteria fue el órgano democrático más importante junto con la asamblea (ekklesiastes). ARISTÓTELES, en Política, define el ciudadano en una democracia como aquel que tiene derecho a ser un miembro del jurado y participante en la asamblea; este análisis, además, coincide con el que aparece de manera específica en su obra sobre la democracia Constitución Ateniense, donde ya en la introducción a la descripción sistemática de la constitución, clasifica la democracia ateniense como aquella donde el poder es ejercido por la Asamblea que adopta decretos y por el Tribunal Popular. 
jurídico e intentar colaborar en la erradicación de las taras político-morales de nuestro tiempo.

La obra de ACKERMAN nos demuestra que el estado anímico de la población es variable, y que se trata de saber si realmente defendemos la idea de que la población de manera directa legisle los contenidos que nos afectan y si estamos dispuestos y preparados para asumir todas las consecuencias y riesgos de tal decisión. Debemos tener siempre en cuenta, como sabía Platón, que la democracia es el gobierno de la opinión y no del conocimiento. En la tarea de la complementación y crítica de ambas teorías hemos querido recuperar elementos del primer concepto de Derecho legítimo de HABERMAS, entendiendo que el proyecto de democracia radical no tiene por qué excluir elementos morales, por mucho que esta validez moral se incluya en los preceptos del Principio del Discurso y en las normas fundamentales universales. Debemos, pues, cuestionarnos si la ciencia jurídica y el Derecho son elementos de conocimiento, de opinión o ambas cosas. La respuesta que demos puede determinar el peso y el campo de actuación de la democracia normativa. Porque, como dice TARR, pertenece a la filosofía discernir y debatir la cuestión de la democracia directa y si ésta es deseable (TARR, 2006).

\section{BIBLIOGRAFÍA}

Abad, A., y MonserRat, J., 2008: «Monism Versus Dualism in the current Spanish System», International Review of Constitutionalism, 8: 1-40.

- 2009: «Habermas and Ackerman, a Synthesis of their Thought applied to the legitimation and codification of Legal Norms», Ratio Juris, 3, in print.

AcKerman, B., 1980: Social Justice in The Liberal State, New Haven, Yale University Press.

- 1983: «What is Neutral about Neutrality?», Ethics, 93 (2):372-390.

— 1989: «Why Dialogue?», The Journal of Philosophy, 86 (1): 5-22.

- 1993: We the people: Foundations, Cambridge, Massachusetts: The Belknap Press of Harvard University Press.

- 1997: «Temporal Horizons of Justice», The Journal of Philosophy, 94 (6): 299-317.

- 2000: «The new separation of power», Harvard Law Review, 113 (3): 633-727.

Ackerman, B., y FishKin, J., 2003: «Deliberation Day», en J. FishKin y P. LASLetT (ed.), 2003: 7-29.

ACKerman, B., y RosenKRANTZ, C., 1991: Fundamentos y alcances del control judicial de constitucionalidad, Madrid, Centro de Estudios Constitucionales.

ARendT, H., 2006: On Revolution, New York, Penguin Classics.

BALKIn, J. M., y Levison, S., 2000: Legal Canons, New York, New York University Press.

BenhabiB, S., 1993: «Models of Public Space: Hannah Arendt, the Liberal Tradition, and Jürgen Habermas», en CalHoun, 1992, 73-97.

Calhoun, C. (ed.), 1992: Habermas and the Public Sphere, The MIT Press.

Dworkin, R., 1978: «Liberalism», en S. HAMPSHIRE (ed.), Public and Private Morality, Cambridge University Press, 113-143.

ElStER, J., 1999: Making Sense of Marx, Cambrigde, Cambridge University Press.

FishKIN, S. J., 1983: «Can There Be a Neutral Theory of Justice?», Ethics, 93 (2): 348-356.

Fishrin, J., y LasLetT, P. (ed.), 2003: Debating Deliberative Democracy, Blackwell. 
Galston, M., y Galston, A. W., 1994: «Contemporary Critics of Liberalism», Ethics, 104 (3): 446-466.

Gargarella, R., 2005: «El constitucionalismo según John Rawls», Revista Araucaria, 14: 3-37.

Gargarella, R., y Ovejero, F., 2001: Razones para el Socialismo, Barcelona, Paidós.

Greenawalt, K., 1994: «Dualism and Its Status», Ethics, 104 (3): 480-499.

HAas, T., 2006: «The Public Sphere as a Sphere of Publics: Rethinking Habermas's Theory of the Public Sphere», Journal of Communication, 54: 178-184.

Habermas, J., 1976: Legitimation Crisis, London, Heinemann Educacional Books.

- 1992: Fäktizitat und Geltung, Beiträge zur Diskurstheorie des Rechts und des demokratischen Rechtsstaats, Frankfurt am Main: Suhrkamp (trad. cast. de M. JiMÉNEZ REDONDO, Facticidad y Validez, sobre el Derecho y el Estado democrático de Derecho en términos de teoría del discurso, Madrid, Trotta, 2001).

Herman, M. H., 1999: The Athenian Democracy in the Age of Demosthenes, London, Bristol Classical Press.

Herzog, D., 1994: «Democratic Credentials», Ethics, 104 (3): 467-479.

HorwiTZ, J. M., 1993: «The Constitution Change: Legal Fundamentality without Fundamentalism», Harvard Law Review, 107: 32-117.

MCILWAn, C. H., 1947: Constitutionalism Ancient and Modern, Cornell UP.

Michelman, F. I., 1999: Brennan and Democracy, Princeton University Press.

MonSERRAT, J., y ABAD, A., 2006: «Las influencias de la obra de Hannah Arendt en el concepto de revolución de B. Ackerman», defendida en el congreso internacional Centenario Hannab Arendt: Entre el presente y el futuro (Universidad Autónoma de Madrid, 29 de noviembre-1 de diciembre de 2006).

Nebelsky, J., 1994: «The Puzzle and Demands of Modern Constitutionalism», Ethics, 104 (3): 500-515.

Posner, A. R., 1992: «Democracy and Dualism», Transition, 56: 68-79.

TARR, A. G., 2006: For the People: Direct Democracy in the State Constitutional tradition, Center of Constitutional Studies, State University of New Jersey.

Thipgen, B. R., y Downing, A. L., 1983: «Liberalism and the neutrality principie», Political Theory, 11 (4): 585-600.

Tushnet, M., 2003: The New Constitutional Order, New Jersey, Princeton University Press.

Vargova, M., 2005: «Democratic Deficits of a Dualist Deliberative Constitutionalism: B. Ackerman and J. Habermas», Ratio Juris, 18 (3): 365-386.

Walker, N., 1993: The Puzzle of modern constitutionalism, Cambridge, The MIT Press.

Zagrebelsky, G., 2005: El Derecho dúctil. Ley, derechos, justicia», Madrid, Trotta. 